\title{
Antitumor activity of Notch-1 inhibition in human colorectal carcinoma cells
}

\author{
WANGDI LIAO ${ }^{1}$, GUOHUA LI $^{1}$, YU YOU $^{1}$, HONGPING WAN $^{2}$, \\ QIONG WU ${ }^{3}$, CHONGWEN WANG ${ }^{1}$ and NONGHUA LV ${ }^{1}$ \\ Departments of ${ }^{1}$ Gastroenterology and ${ }^{2}$ Pathology, The First Affiliated Hospital, Nanchang University; \\ ${ }^{3}$ Department of Hematology, The Second Affiliated Hospital of Nanchang University, \\ Jiangxi Medical Molecule Key Laboratory, Nanchang, Jiangxi 330006, P.R. China
}

Received June 12, 2017; Accepted December 12, 2017

DOI: $10.3892 /$ or.2017.6176

\begin{abstract}
This study investigated the roles of Notch-1 in colorectal carcinoma, to assess the mechanisms. The expression of Notch-1 and its ligand-Jagged1 was detected in human colorectal carcinoma, colorectal adenoma, paracancerous tissue and normal colorectal tissue by immunohistochemistry. Colorectal carcinoma cell lines were utilized to confirm the expression of Notch-1 in colorectal carcinoma cells. Lentiviral- encoding Notch-1-siRNA, as well as Notch-1 inhibitor was employed to silence Notch-1 expression and to inhibit Notch-1 activity in HT29 cells, respectively. As evidenced, Notch-1 and Jagged1 were highly expressed in colorectal carcinoma and colorectal adenoma tissues, compared with those in paracancerous tissue and normal colorectal tissue. However, the expression of Notch-1 and Jagged1 was comparable in colorectal carcinoma and colorectal adenoma tissues, and in paracancerous and normal colorectal tissues. After screening colorectal carcinoma cell lines, Notch-1 was extensively expressed in COLO 205, HT29, SW480 and SW1116 cells, but slightly expressed in LoVo cells. Subsequently, HT29 cell line was selected to investigate the roles of Notch-1 in tumor cell growth and apoptosis. Lenti-viral encoding Notch-1 siRNA significantly decreased Notch-1 expression, inhibited cell growth, arrested the cell cycle at G1 phase and promoted apoptosis. These effects were further confirmed by the Notch-1 inhibitor DAPT. Additionally, we evidenced that Notch-1 silence promoted P21 and PUMA expression in HT29 cells. Taken together, Notch-1 is an oncogene in colorectal carcinoma and the inhibition of Notch-1 could delay the cell growth and promote apoptosis in colorectal cancer.
\end{abstract}

Correspondence to: Dr Nonghua Lv, Department of Gastroenterology, The First Affiliated Hospital, Nanchang University, 17 Yongwai Street, Nanchang, Jiangxi 330006, P.R. China

E-mail: lunonghua@163.com

Key words: Notch signaling pathway, colorectal carcinoma, apoptosis, DAPT

\section{Introduction}

Colorectal carcinoma is a common malignant tumor of the digestive system. In North America and Western Europe, colorectal carcinoma is the third leading cause of cancer death (1). Recently, the incidence and mortality of colorectal cancer in China are showing a rising trend (2). However, the effective therapeutic method for colorectal carcinoma is still lacking.

Notch signaling pathway is a highly-conserved intercellular signal transduction pathway, which is composed of Notch receptor, Notch ligand (DSL protein) and transcription factor CSL (DNA binding protein). Notch signaling pathway regulates cell differentiation, proliferation and apoptosis, and plays important roles in embryonic development and cell fate determination (3). Notch signaling pathway not only plays an important role in normal cell differentiation, but also participates in the occurrence and development of tumors (4). The expression of Notch receptors and ligands, and the aberrant activation of Notch signaling pathway have been found in a variety of malignant tumors $(5,6)$. However, Notch signaling pathway and its role in tumorigenesis are extremely complex. Notch signaling pathway plays a role in promoting tumorigenesis in most cases $(7,8)$, but functions as tumor suppressor in a few cases. The discrepancy is likely caused by that Notch-1 which was detected in different tumor stages $(9,10)$. Additionally, Notch expression may be associated with the cell microenvironment, tissue type and determines cell proliferation, differentiation, self-renewal or apoptosis $(11,12)$. Notch signaling pathway has been extensively investigated in tumor therapy (13). DAPT, a $\gamma$-secretase inhibitor can block Notch receptor/ligand binding enzyme cleavage, thereby blocking the Notch signaling. Therefore, DAPT has been used in clinical trials to treat cancers (14).

There are a variety of Notch receptors and ligands expressed in the gut tissue $(15,16)$. Ngn3, HES1 and Math1, three regulators of Notch signaling pathway have intestinal phenotype. Transgenic methods further confirmed that Notch directly regulated the fate of intestinal epithelial cells (17). van Es et al (18) inhibited the Notch signal by using the $\gamma$-secretase inhibitor, and the differentiation of colon adenoma cells in mice recovered. Nevertheless, the relationship between Notch and colorectal cancer is not clear. In this study, we 
screened the expression of Notch-1 in colorectal cancer tissue and cancer cell lines, and investigated the functions of Notch-1 in colorectal biological activities.

\section{Materials and methods}

Colorectal cancer tissues and cell lines. Colorectal carcinoma, colorectal adenoma and paracancerous tissues and normal colorectal tissues were obtained from the First Affiliated Hospital of Nanchang University. This study was approved by the Ethics Committee of Nanchang University. Colorectal cancer cell lines (COLO 205, HT29, SW480 and SW1116) were gifted by Digestion Institute of Nanfang Hospital. LoVo cells were obtained from Institute of Biochemistry and Cell Biology, Chinese Academy of Sciences (China).

Cell culture and transfection. Colorectal cancer cell lines (COLO 205, HT29, SW480, SW1116 and LoVo) were cultured in Dulbecco's minimum essential medium (DMEM) (Gibco, Grand Island, NY, USA) supplemented with $10 \%$ fetal bovine serum (FBS) and $100 \mathrm{U} / \mathrm{ml}$ penicillin-streptomycin (Sigma, Ronkonkoma, NY, USA) in $5 \% \mathrm{CO}_{2}$ at $37^{\circ} \mathrm{C}$. Cell confluence at $50-70 \%$ was applied in the following experiments. The cells were divided into three groups: non-RNAi group (NR), negative control group (NC) and RNAi group (R). pSiRNA-Notch-1 and empty vector pSilencer 5.1-H1 Retro (Shanghai GenePharma, Shanghai, China) were transfected by Lipofectamine 2000 and packaged into viruses.

DAPT treatment. HT29 cells were treated by DAPT $(6.25-50 \mu \mathrm{M})$ (Sigma) for 1, 2, 3 and 4 days, respectively. After treatments, the cell proliferation and apoptosis were detected. DAPT was dissolved in $0.2 \%(\mathrm{v} / \mathrm{v})$ DMSO and a similar concentration of DMSO was applied as negative control. Proliferation was detected by 3-(4,5-dimethyl-2-thiazolyl)-2,5-diphenyl-2-H-tetrazolium bromide (MTT) assay. The cell cycle and apoptosis were detected by flow cytometry and terminal deoxynucleotidyl transferase dUTP nick end labeling (TUNEL) assay.

MTT assay. Cells were seeded in 96-well plates. When cell confluence reached 50-70\%, $100 \mu \mathrm{l}$ virus supernatant was added to knock down Notch-1 expression. After transfection for 1, 2, 3 and 4 days, MTT assay was applied to detect the cell proliferation as previously described (15). The optical density (OD) was determined by Microplate Reader (BioTek, Winooski, VT, USA) at $570 \mathrm{~nm}$.

Flow cytometry. When cell confluence reached 50-70\%, $100 \mu \mathrm{l}$ virus supernatant was added to knock down Notch-1 expression. After transfection for $48 \mathrm{~h}$, the cells were collected for Annexin V-FITC/PI staining (Beyotime, Ningbo, China) and apoptosis was detected within $1 \mathrm{~h}$ by FACSCalibur (BD Biosciences, Franklin Lakes, NJ, USA).

After transfection for $48 \mathrm{~h}$, the cells were collected for PI staining and cell cycle distribution was assessed by FACSCalibur (BD Biosciences) within $1 \mathrm{~h}$ after staining.

TUNEL assay. TUNEL assay was conducted according to the instruction of DeadEnd ${ }^{\mathrm{TM}}$ Colorimetric TUNEL system (Promega, Madison, WI, USA).
Immunohistochemical and immunocytochemical staining. Cancer tissues were fixed in 10\% formaldehyde and embedded in paraffin. Sections (3-5 $\mu \mathrm{m}$ ) were continuously sliced. After dewaxing by xylene, the tissues were dehydrated in $70,75,80$, 85 and $95 \%$ gradient alcohol. Hydrogen peroxide $(3 \%$ ) was applied to repair the antigen. The mounted cells were fixed in acetone. The non-specific staining was blocked by goat serum at $4^{\circ} \mathrm{C}$ overnight. Immunostaining of histological sections was performed using monoclonal antibodies against Notch-1 (1:200, ab52627; Abcam, Cambridge, MA, USA) and Jagged1 (1:200, ab7771; Abcam) overnight at $4^{\circ} \mathrm{C}$ followed by a 30 -min incubation with secondary antibody (Dako, Carpinteria, CA, USA) and visualization with 3,3'-diaminobenzidine (DAB) for $3 \mathrm{~min}$. PBS was employed as the negative control for the primary antibody. The positive staining was analyzed by ImageJ software.

Reverse-transcript PCR. Total RNA was extracted from tissues or cell lines using TRIzol reagent (Thermo Fisher Scientific, Waltham, MA, USA). RNA concentrations were determined spectrophotometrically, and $1 \mu \mathrm{g}$ total RNA was reverse transcribed using an avian myeloblastosis virus reverse-transcriptase kit (Promega). The expression of Notch-1, p53 upregulated modulator of apoptosis (PUMA), p21 and $\beta$-actin in mRNA was detected. PCR primers used were as follows: Notch-1-F, CTCCCCGTTCCAGCAGTCTC and Notch-1-R, CAGCCACTCGCATTGACCAT; PUMA-F, GATGGCGGACGACCTCAAC and PUMA-R, ACGGGCA CCAGCACAACA; p21-F, TTGATTAGCAGCGGAACA and p21-R, TACAGTCTAGGTGGAGAAACG; $\beta$-actin-F, AAGG AAGGCTGGAAGAGTGC and $\beta$-actin-R, CTACAATGAG CTGCGTGTGG.

The amplification reactions were carried out within a PCR system (Applied Biosystems), with initial hold step $\left(95^{\circ} \mathrm{C}\right.$ for $4 \mathrm{~min})$ and 40 cycles of a PCR $\left(95^{\circ} \mathrm{C}\right.$ for $40 \mathrm{sec}, 54^{\circ} \mathrm{C}$ for $30 \mathrm{sec}$ and $72^{\circ} \mathrm{C}$ for $30 \mathrm{sec}$ ). The expression of target genes was normalized to $\beta$-actin.

Western blotting. Protein was extracted from cell lines for western blotting as previously described (19). The anti-Notch-1 antibody (1:200, ab52627; Abcam) was incubated overnight at $4{ }^{\circ} \mathrm{C}$. After washing with $0.1 \%$ PBST $(0.1 \%$ Tween-20), membranes were probed with anti-mouse or anti-rabbit horseradish peroxidase secondary antibodies $(1: 100$; cat. nos. ab131368 and ab191866; Abcam) at room temperature for $2 \mathrm{~h}$. Enhanced Chemiluminescent reagent kit (GE Healthcare Life Sciences, Chalfont, UK) was applied to assist the staining. The blots were scanned by ChemiDoc ${ }^{\mathrm{TM}}$ XRS (Bio-Rad Laboratories, Hercules, CA, USA) and grey density was analyzed by Quantity One v4.62.

Statistical analysis. Data are presented as means \pm standard deviations. One-way analysis of variance with post hoc Bonferroni test for multiple comparisons was performed. Differences were considered significant at $\mathrm{P}<0.05$.

\section{Results}

Expression of Notch-1 and Jagged1. Initially, we detected the expression of Notch-1 and its ligand-Jagged1 in colorectal 


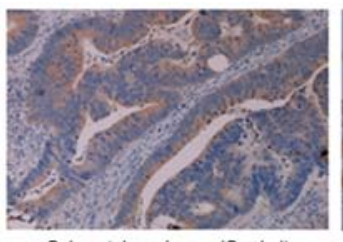

Colorectal carcinoma (Grade I)

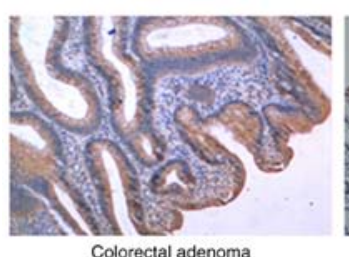

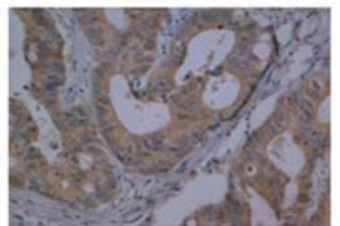

Colorectal carcinoma (Grade II)

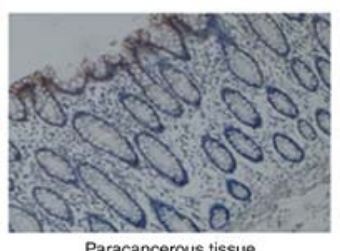

Paracancerous tissue

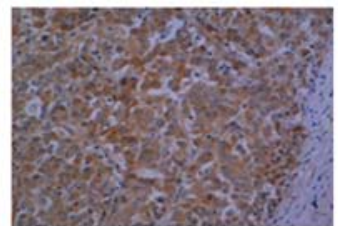

Colorectal carcinoma (Grade III)

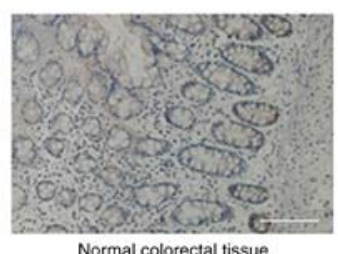

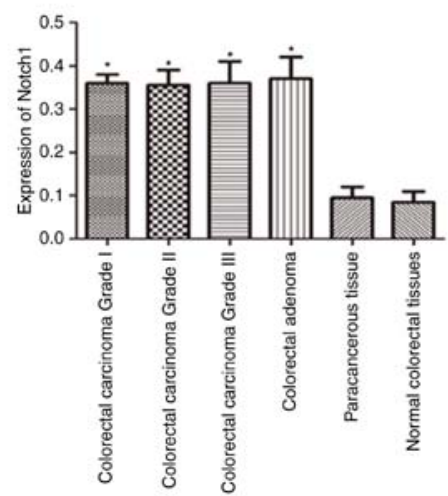

Figure 1. Notch-1 expression in colorectal carcinoma (grade I, II and III), colorectal adenoma, paracancerous tissue and normal colorectal tissue. Upper panel: representative images. Lower panel: quantification data. ${ }^{*} \mathrm{P}<0.05$ compared with normal colorectal tissue. Scale bar, $100 \mu \mathrm{m}$.

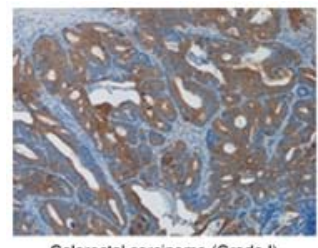

Colorectal carcinoma (Grade I)

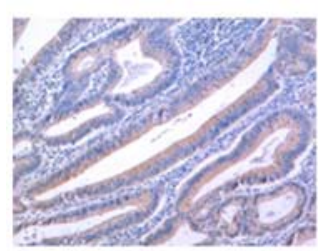

Colorectal adenoma

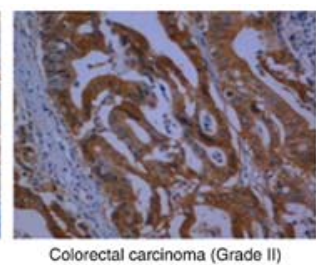

Colorectal carcinoma (Grade II)

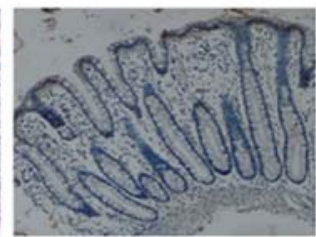

Paracancerous tissue

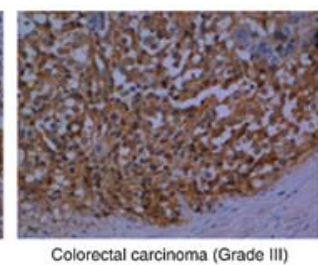

Colorectal carcinoma (Grade III)

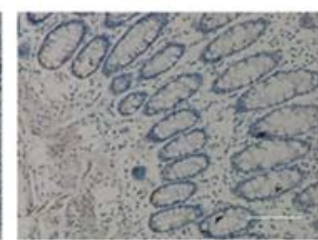

Normal colorectal tissue

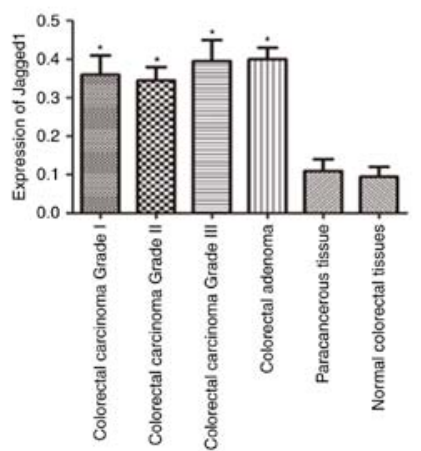

Figure 2. Jagged1 expression in colorectal carcinoma (grade I, II and III), colorectal adenoma, paracancerous tissue and normal colorectal tissue. Upper panel: representative images. Lower panel: quantification data. ${ }^{*} \mathrm{P}<0.05$ compared with normal colorectal tissue. Scale bar, $100 \mu \mathrm{m}$.

carcinoma, colorectal adenoma and carcinoma-adjacent tissues and normal colorectal tissues. As shown in Figs. 1 and 2, the expression of Notch-1 (Fig. 1) and Jagged1 (Fig. 2) in colorectal carcinoma and colorectal adenoma was obviously 

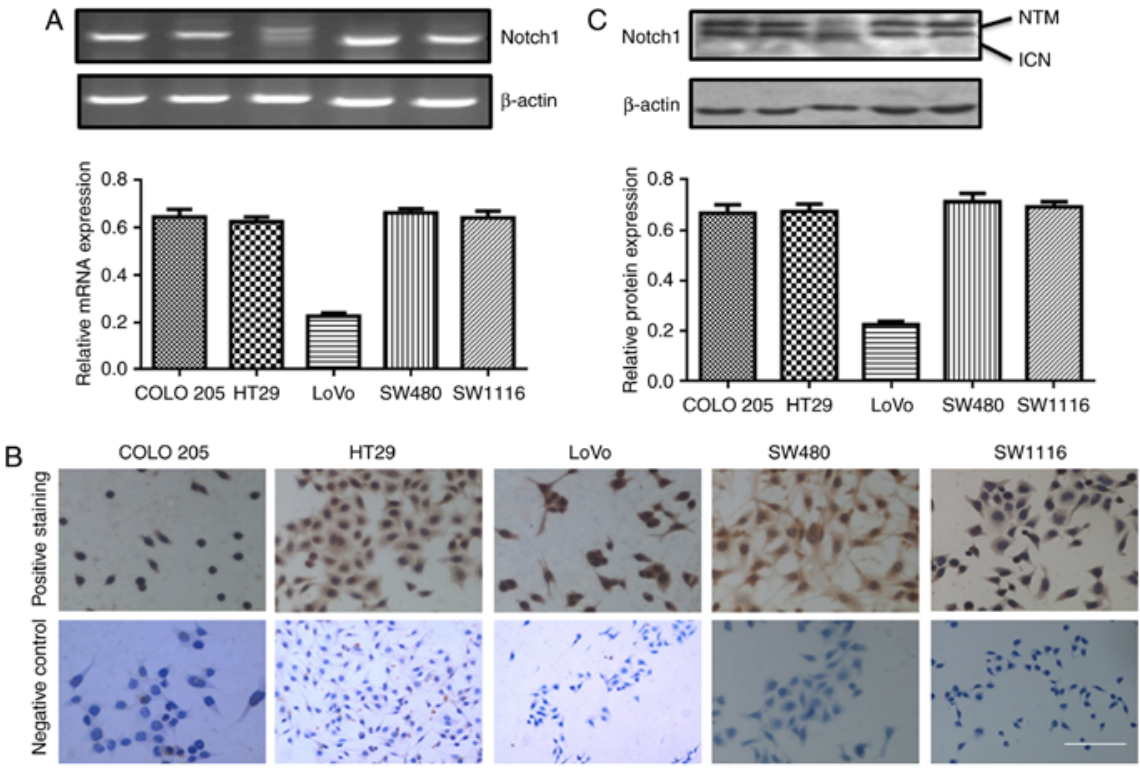

Figure 3. Notch-1 expression in colorectal carcinoma cells. (A) RT-PCR of Notch-1 expression. (B) Immunocytochemical staining of Notch-1, Scale bar, $100 \mu \mathrm{m}$. (C) Western blotting of Notch-1 expression. NC, negative control.
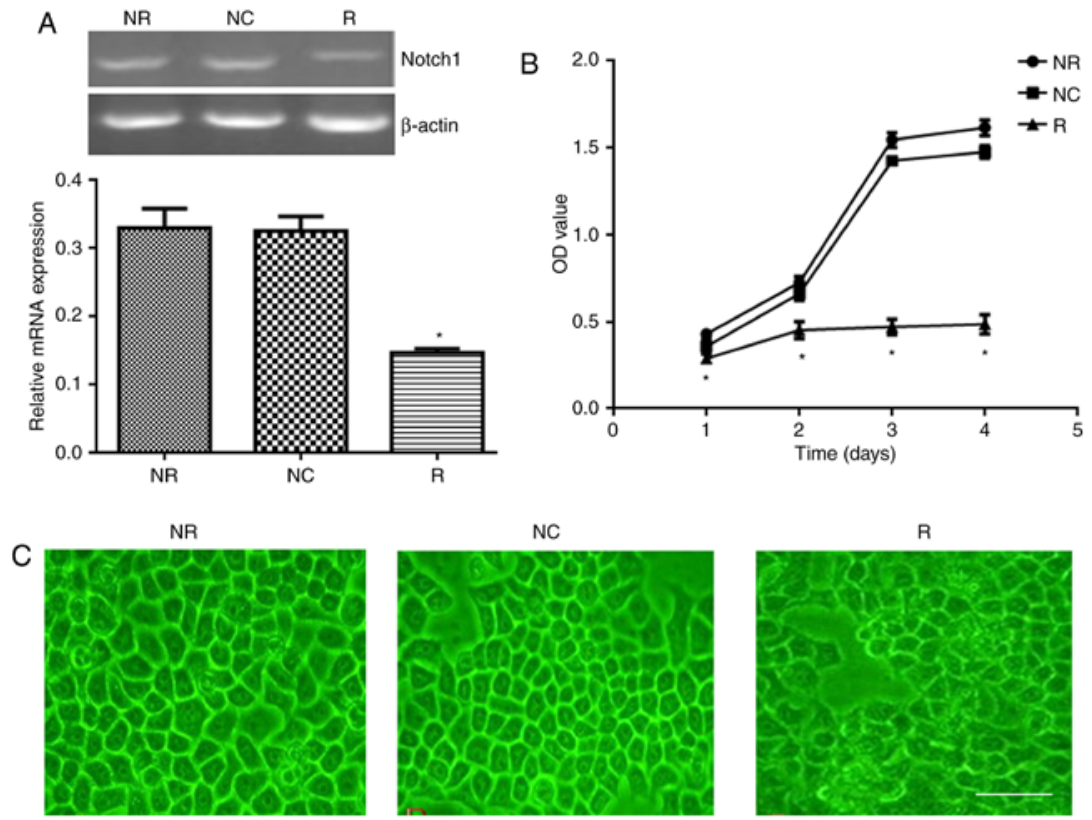

Figure 4. Notch-1 silence inhibits cell proliferation in HT29 cells. (A) RT-PCR detecting Notch-1 expression. (B) Cell proliferation. (C) Cell morphology observed by microscopy. Scale bar, $50 \mu \mathrm{m}$. "P $<0.05$ compared with NR. NR, non-RNAi group; NC, negative control group, R, RNAi group.

higher than those in carcinoma-adjacent tissues and normal colorectal tissues. However, there was no significant difference between carcinoma-adjacent tissues and normal colorectal tissues regarding Notch-1 and Jagged1 expression.

We also detected Notch-1 mRNA expression in COLO205, HT29, LoVo, SW480 and SW1116 cells by RT-PCR. As shown in Fig. 3A, Notch-1 expression was relatively higher in COLO 205, HT29, SW480 and SW1116 cells, compared with LoVo. Immunohistochemical staining showed that Notch-1 was expressed in all five colorectal carcinoma cells (Fig. 3B). Two sub-types of Notch-1-4 exist: Notch transmembrane fragment (NTM) and intracellular domain of Notch (ICN). We found two bands of Notch-1 in all the five cell lines. Especially,
Notch-1 was highly expressed in COLO 205, HT29, SW480 and SW1116 cells (Fig. 3C).

pSiRNA-Notch-1 inhibits Notch-1 expression and cell proliferation in HT29 cells. In the next part of experiments, we designed pSiRNA-Notch-1 and transferred the vector into viruses. The viral supernatant was collected to transfect HT29 cells. Our data showed that viral pSiRNA-Notch-1 significantly reduced Notch-1 expression in HT29 cells compared with NC group (Fig. 4A). The cell proliferation after viral transfection was detected. As shown in Fig. 4B, viral pSiRNA-Notch-1 significantly inhibited the cell proliferation compared with NC group. The morphology of the cells was also observed. As 


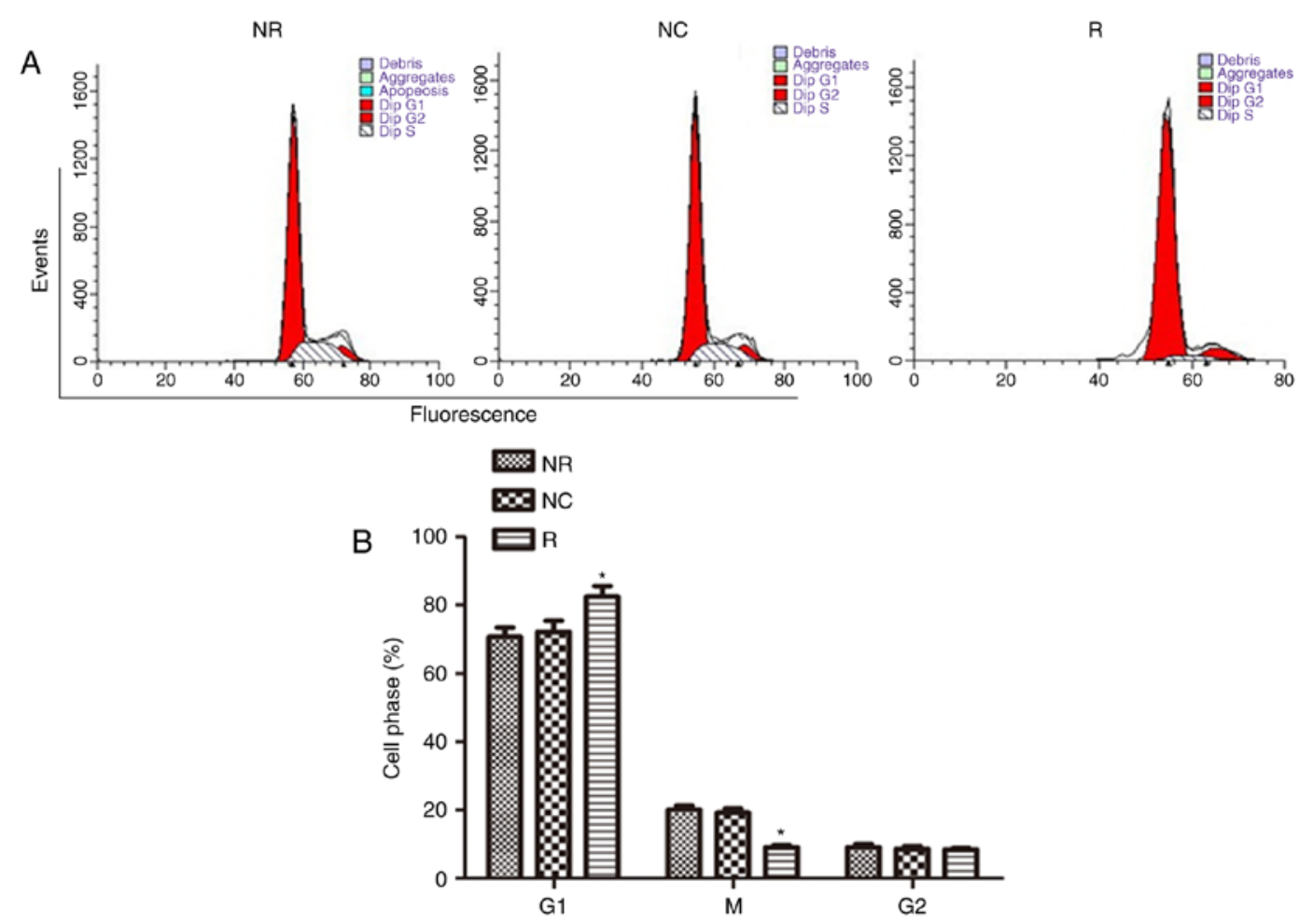

Figure 5. Notch-1 silence arrests cell cycle at G0/G1 phase in HT29 cells. (A) PI staining detected by flow cytometry. (B) Quantification data. ${ }^{*} \mathrm{P}<0.05$ compared with NR. NR, non-RNAi group; NC, negative control group, R, RNAi group.
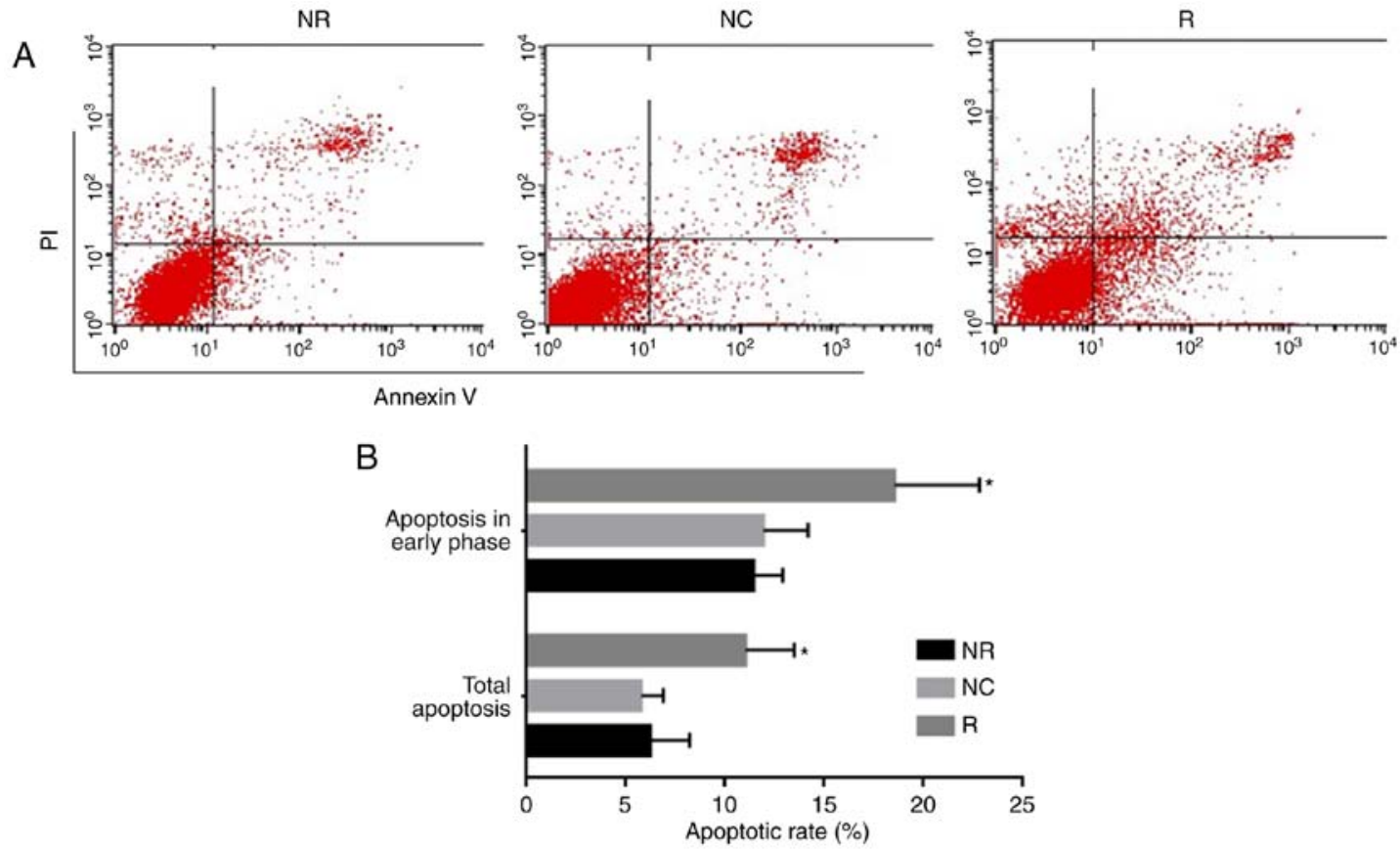

Figure 6. Notch-1 silence promotes apoptosis in HT29 cells. (A) Annexin V/PI staining detected by flow cytometry. (B) Quantification data. ${ }^{*} \mathrm{P}<0.05$ compared with NR. NR, non-RNAi group; NC, negative control group, R, RNAi group.

shown in Fig. 4C, the cells in NR and NC groups showed clear boundary, while the cells in viral pSiRNA-Notch-1 group showed diffused cell boundary. Abundant dead cells were observed after Notch-1 knockdown.

pSiRNA-Notch-1 arrests the cells at G1 phase and promotes apoptosis. Cell cycle was detected by flow cytometry after
PI staining. As shown in Fig. 5A and B, Notch-1 knockdown significantly arrested the cell cycle at G1 phase (vs NC, P<0.05) (NR: 70.75\%; NC: 72.18\%; R: 82.43\%). Apoptosis was detected by TUNEL assay and flow cytometry. As shown in Fig. 6, Notch-1 knockdown not only promoted apoptosis in early phase, but also total apoptosis. These data were further confirmed by TUNEL assay (Fig. 7). 

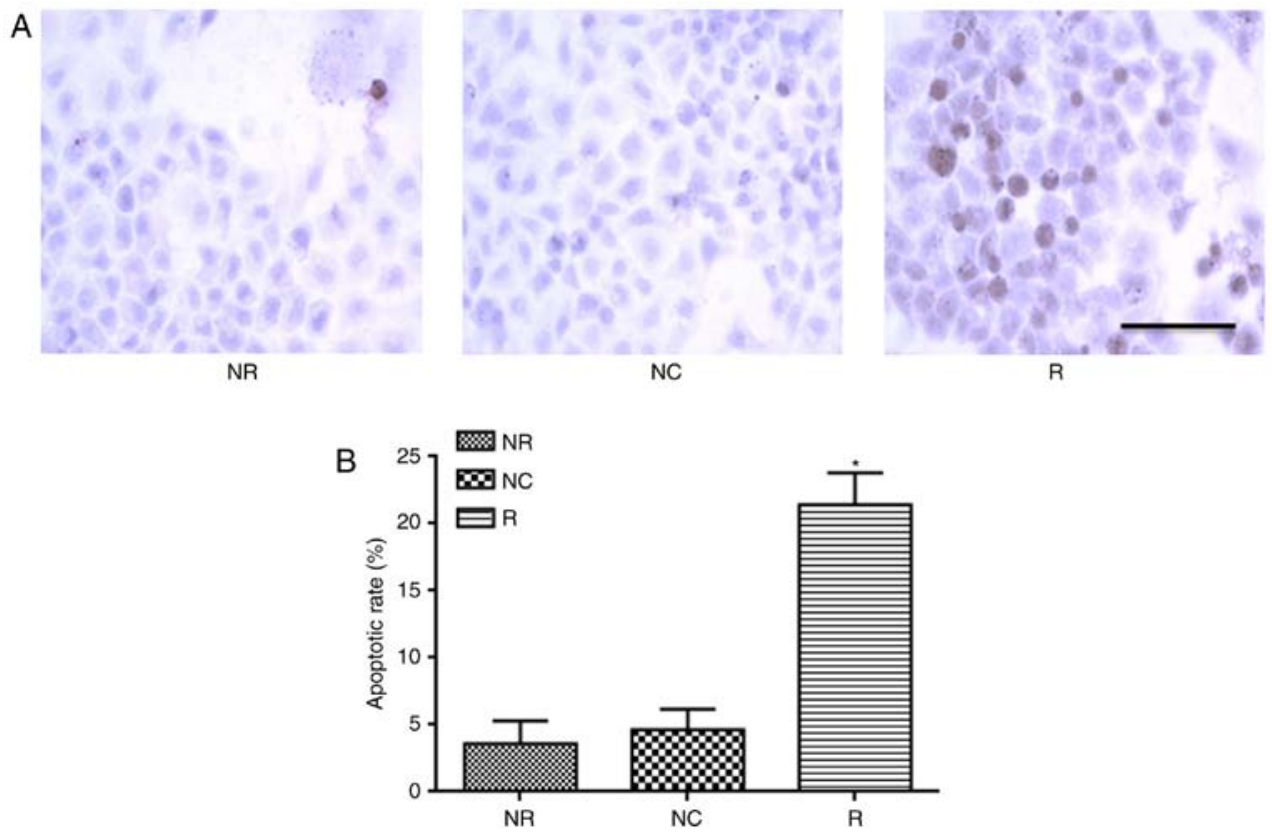

Figure 7. Notch-1 silence promotes apoptosis in HT29 cells. (A) TUNEL staining. (B) Quantification data. "P<0.05 compared with NR. Scale bar, $50 \mu \mathrm{m}$. $\mathrm{NR}$, non-RNAi group; $\mathrm{NC}$, negative control group, R, RNAi group.
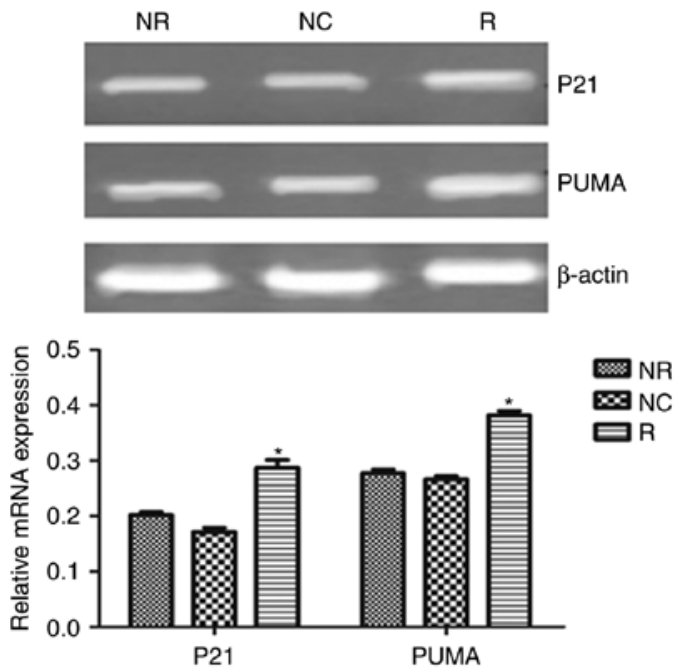

Figure 8. Notch-1 silence promotes expression of p21 and PUMA in HT29 cells. ${ }^{\mathrm{P}}<0.05$ compared with NR. NR, non-RNAi group; NC, negative control group, R, RNAi group.

pSiRNA-Notch-1 promotes 221 and PUMA expression. We also detected apoptosis-related gene expression. As shown in Fig. 8, Notch-1 knockdown significantly increased p21 and PUMA expression in mRNA level.

DAPT inhibits cell proliferation, arrests cell cycle at G1 phase and promotes apoptosis. We also applied different concentrations of DAPT in HT29 cells. As shown in Fig. 9A, DAPT at the concentration range of 6.25-50 $\mu \mathrm{M}$ inhibited the cell proliferation in a concentration-dependent manner. After incubation for 4 days, DAPT at 12.5-50 $\mu \mathrm{M}$ significantly inhibited cell proliferation compared with control. DAPT treatment $(50 \mu \mathrm{M})$ for $48 \mathrm{~h}$ impaired cell morphology and structure (Fig. 9B). TUNEL assay showed that DAPT promoted apoptosis at the
Table I. DAPT triggers apoptosis in HT29 cells after administration for $48 \mathrm{~h}$.

\begin{tabular}{lccc}
\hline Groups & Control & $25 \mu \mathrm{M}$ DAPT & $50 \mu \mathrm{M}$ DAPT \\
\hline Apoptosis (\%) & $4.22 \pm 2.51$ & $17.61 \pm 2.82^{\mathrm{a}}$ & $25.34 \pm 5.28^{\mathrm{a}}$
\end{tabular}

${ }^{\mathrm{a}} \mathrm{P}<0.05$ compared with control.

concentrations of 25 and $50 \mu \mathrm{M}$ (Fig. 9C and Table I). Compared with control, there was significant difference regarding apoptotic rate in 25 and $50 \mu \mathrm{M}$ DAPT groups. DAPT-induced apoptosis in HT29 cells were also confirmed by flow cytometry after Annexin V-FITC/PI double staining (Fig. 9D). DAPT treatment (25 and $50 \mu \mathrm{M}$ ) for $48 \mathrm{~h}$ not only promoted apoptotic rate in early phase, but also total apoptotic rate compared with control (Fig. 9D and Table II). Additionally, we found DAPT arrested the cell cycle at G1 phase after 48-h treatment (Fig. 9E and Table III).

\section{Discussion}

Notch signaling pathway plays key roles in determining cell fate in multicellular organisms $(20,21)$. Transgenic animal model showed that the fate of intestinal epithelial cells was directly regulated by Notch (22). However, the relationship between the abnormal expression of Notch-1 and colorectal cancer is not clear. Our study showed that Notch-1 expression in colorectal cancer was significantly higher than those of paracancerous tissues and normal colorectal tissues, which was consistent with a previous study (23). In this study, we also found for the first time that the expression of Jagged 1 in colorectal cancer was increased compared with paracancerous tissues and normal colorectal tissues. These results suggest 

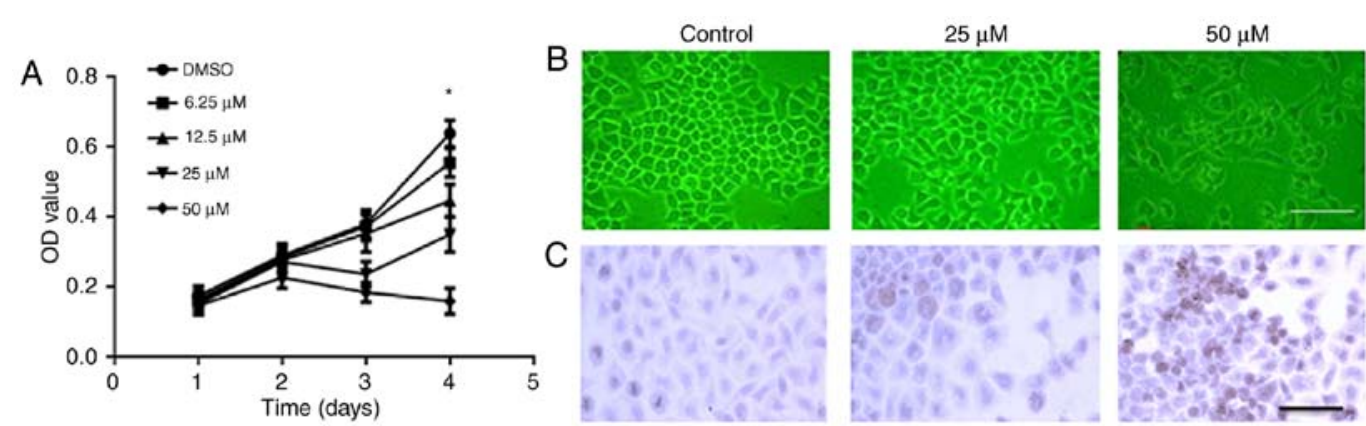

D
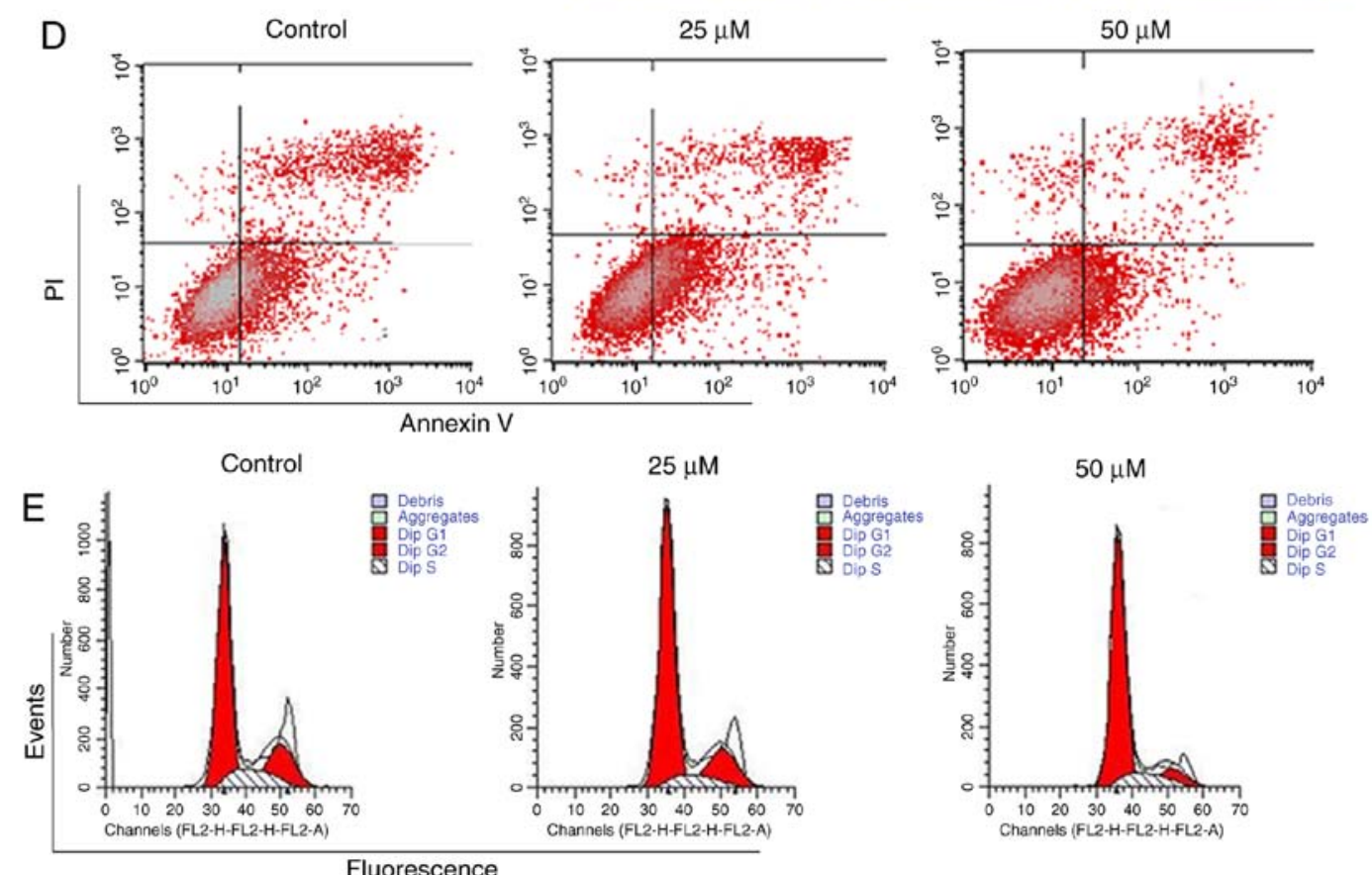

Figure 9. DAPT suppresses cell proliferation in HT29 cells. (A) MTT assay. (B) Cell morphology. (C) TUNEL assay. (D) Annexin V/PI staining detected by flow cytometry. (E) PI staining detected by flow cytometry. ${ }^{\mathrm{P}}<0.05$ compared with control. Scale bar, $50 \mu \mathrm{m}$.

Table II. DAPT triggers apoptosis in HT29 cells after administration for $48 \mathrm{~h}$.

\begin{tabular}{lccc}
\hline Groups & Control & $25 \mu \mathrm{M}$ DAPT & $50 \mu \mathrm{M}$ DAPT \\
\hline $\begin{array}{l}\text { Apoptosis in } \\
\text { early phase (\%) }\end{array}$ & $12.44 \pm 0.99$ & $25.30 \pm 0.74^{\mathrm{a}}$ & $32.05 \pm 0.80^{\mathrm{a}}$ \\
Total apoptosis (\%) & $20.48 \pm 1.57$ & $36.43 \pm 1.42^{\mathrm{a}}$ & $43.24 \pm 1.67^{\mathrm{a}}$
\end{tabular}

${ }^{\mathrm{a}} \mathrm{P}<0.05$ compared with control.

that Notch-1 signaling pathway is involved in the pathogenesis of colorectal cancer. However, the expression was not related to the cancer grades, suggesting that Notch signaling is involved in carcinogenesis of colorectal cancer even at the early phase. A large number of studies have shown that Notch signaling pathway, especially Notch-1, is closely related to tumorigenesis (24-29). Immunocytochemical staining showed that Notch-1 was expressed in all five colorectal cancer cells. These results were confirmed by RT-PCR and western blotting methods. Interestingly, Notch-1 is highly expressed in HT29, SW480, COLO205 and SW1116, while
Table III. DAPT causes cell cycle arrest in HT29 cells after administration for $48 \mathrm{~h}$.

\begin{tabular}{lccc}
\hline Groups & Control & $25 \mu \mathrm{M}$ DAPT & $50 \mu \mathrm{M}$ DAPT \\
\hline G1 phase (\%) & $64.813 \pm 2.525$ & $71.517 \pm 1.562^{\mathrm{a}}$ & $75.797 \pm 1.251^{\mathrm{a}}$ \\
S phase (\%) & $18.456 \pm 1.364$ & $12.369 \pm 1.014$ & $10.089 \pm 0.897$ \\
G2 phase (\%) & $16.731 \pm 1.145$ & $16.114 \pm 1.125$ & $14.114 \pm 0.974$ \\
\hline
\end{tabular}

${ }^{a} \mathrm{P}<0.05$ compared with control.

LoVo cells showed relatively low expression. The in vitro data further confirmed that Notch signaling is related to the development of colorectal cancer.

Gene therapy is a hot topic in the research of tumor therapy. RNA interference (RNAi) is a promising method in the study of gene function (30). RNAi technology is simple, fast and effective and could specifically inhibit the expression of target genes. Therefore, this technique is now a powerful tool to study gene function, but also provides new technology for the treatment of cancers (31). In our study, PSilencer 5.1-H1 Retro source was derived from murine stem cell virus (MSCV), 
a retroviral vector carrying siRNA fragment. The total length was $6253 \mathrm{bp}$, and the promoter is started by $\mathrm{H} 1$, with Bam HI and HindIII restriction sites. Therefore, the viruses can effectively transfect mammalian cells. Due to the resistance to puromycin, it is convenient to use the cells to express the recombinant DNA. In order to clarify the role of Notch signaling pathway performed in the proliferation of colorectal cancer, we selected HT29 cells and knocked down Notch-1 expression. Additionally, Notch signaling pathway inhibitor DAPT was also utilized to confirm the effects of Notch signaling pathway on proliferation of colorectal cancer cells. MTT assay showed that the growth and proliferation of HT29 cells were suppressed after Notch-1 knockdown, accompanied with cell morphological changes of cell death. These results suggest that Notch-1 signaling can promote the growth and proliferation of colorectal cancer cells. DAPT is a chemical molecule that specifically inhibits the activity of $\gamma$-secretase. Therefore, DAPT can block the cleavage of Notch receptor/ligand binding and inhibit all Notch signals. We treated HT29 cells with DAPT and results showed that DAPT treatment could slow down HT29 cell proliferation in a concentration- and time-dependent manner. Interestingly, a small dose of DAPT could promote chemotherapy in colon cancer cells (32). Mechanically, Notch-1 knockdown or inhibition of Notch signaling pathway is able to inhibit cell cycle and arrest the cell cycle at G0/G1 phase, and induce apoptosis in other tumors such as malignant melanoma and pancreatic cancer. In our study, we also revealed that Notch signaling pathway inhibition might suppress the growth and proliferation of colorectal cancer cells by affecting cell cycle and inhibiting cell apoptosis.

p21 protein has function in arresting cell cycle by inhibiting Cyclin-dependent kinase (CDK) complexes, such as cyclin A-CDK2, cyclin D-CDK4/CDK6 and cyclin E/CDK2. p21 expression can block cell cycle at G1 phase, G2 phase or $\mathrm{S}$ phase (33). We used RT-PCR method to detect the effects of Notch-1 interference on the expression of p21 mRNA in HT29 cells. The results showed that Notch-1 silence increased expression of $\mathrm{p} 21$. Therefore, we hypothesized that Notch-1 knockdown may lead to cell cycle arrest by inhibiting the expression of $\mathrm{p} 21$, thereby inhibiting the growth of colorectal cancer cells. Sjölund et al (34) also found that Notch-1 knockout can inhibit the growth of renal cell carcinoma in vitro and in vivo, and arrest the cell cycle at G0/G1 phase. p21 not only inhibits the cell cycle, but also promotes apoptosis in some conditions. For example, trichostatin A can upregulate p21 to induce apoptosis of osteoclasts (35). Zoli et al found that the expression of p21 was upregulated and was positively correlated with apoptosis of breast cancer treated with adriamycin paclitaxel combined with 5-fluorouracil (36). Therefore, we assumed that Notch-1 signaling pathway activation in colorectal cancer cells may suppress apoptosis of HT29 cells by downregulating the expression of $\mathrm{p} 21$.

PUMA is a pro-apoptotic gene and one member of apoptosis-related proteins in the Bcl-2 family, through its interaction with Bcl-2/ Bcl-XL (37). Previous results showed that transfection of PUMA into human melanoma cell line can lead to cell apoptosis and death in p53-dependent or -independent pathways (38). Our study found that the expression of
PUMA was upregulated after Notch-1 interference in HT29 cells. These results implicated that the interference of Notch-1 could promote apoptosis by upregulating the expression of PUMA. Stylianou et al found that chemotherapy by mitoxantrone was related to Notch-regulated PUMA and apoptosis in breast cancer cell lines (39). These results suggest that Notch signaling can regulate the cell cycle and cell apoptosis by regulating the p21 and PUMA genes, and thus plays a role in promoting cancer in colorectal cancer.

In our study, we verified Notch-1 expression in cancer tissue and cell lines. In the next part of experiments, HT29 cells were selected because of its high expression of Notch-1. Several siRNAs were designed to knock down Notch-1 and one optimal siRNA was packaged in the virus. Moreover, the role of Notch-1 was further confirmed by DAPT. In future studies, more colorectal cancer cell lines will be selected to clarify the mechanisms.

In conclusion, Notch-1 is an oncogene in colorectal cancer cells. Its overexpression is related to the cancer development. Moreover, genetic method to knock down Notch-1 or Notch signaling pathway inhibitor can effectively suppress the growth and proliferation of colorectal cancer cells, and promote cell apoptosis. These results indicate that Notch-1 could serve as a therapy target for colorectal cancer.

\section{Acknowledgements}

This study was supported by Natural Science Foundation of Jiangxi Province (20122BAB205018) and Foundation of Jiang'xi Educational Committee (GJJ11328).

\section{References}

1. Eadens MJ and Grothey A: Curable metastatic colorectal cancer. Curr Oncol Rep 13: 168-176, 2011.

2. Li L, Li YM, Zhou P, Wang XS, Wang GY, Zhao XH, Cui BB, Ren YL, Dong XS and Chen ZQ: Abnormal expression of p190RhoGAP in colorectal cancer patients with poor survival. Am J Transl Res 8: 4405-4414, 2016.

3. Huang T, Zhou Y, Cheng AS, Yu J, To KF and Kang W: NOTCH receptors in gastric and other gastrointestinal cancers: Oncogenes or tumor suppressors? Mol Cancer 15: 80, 2016.

4. Zhang M, Biswas S, Qin X, Gong W, Deng W and Yu H: Does Notch play a tumor suppressor role across diverse squamous cell carcinomas? Cancer Med 5: 2048-2060, 2016.

5. Tarulli GA, Butler LM, Tilley WD and Hickey TE: Bringing androgens up a NOTCH in breast cancer. Endocr Relat Cancer 21: T183-T202, 2014.

6. Leong KG and Karsan A: Recent insights into the role of Notch signaling in tumorigenesis. Blood 107: 2223-2233, 2006.

7. Koch U and Radtke F: Notch and cancer: A double-edged sword. Cell Mol Life Sci 64: 2746-2762, 2007.

8. Radtke F and Raj K: The role of Notch in tumorigenesis: Oncogene or tumour suppressor? Nat Rev Cancer 3: 756-767, 2003.

9. Talora C, Sgroi DC, Crum CP and Dotto GP: Specific downmodulation of Notch1 signaling in cervical cancer cells is required for sustained HPV-E6/E7 expression and late steps of malignant transformation. Genes Dev 16: 2252-2263, 2002.

10. Collins BJ, Kleeberger W and Ball DW: Notch in lung development and lung cancer. Semin Cancer Biol 14: 357-364, 2004.

11. Li S, Hu H, He Z, Liang D, Sun R and Lan K: Fine-tuning of the Kaposi's sarcoma-associated herpesvirus life cycle in neighboring cells through the RTA-JAG1-Notch pathway. PLoS Pathog 12: e1005900, 2016.

12. Li L, Grausam KB, Wang J, Lun MP, Ohli J, Lidov HG, Calicchio ML, Zeng E, Salisbury JL, Wechsler-Reya RJ, et al: Sonic Hedgehog promotes proliferation of Notch-dependent monociliated choroid plexus tumour cells. Nat Cell Biol 18: 418-430, 2016. 
13. Cigliano A, Wang J, Chen X and Calvisi DF: Role of the Notch signaling in cholangiocarcinoma. Expert Opin Ther Targets 21: 471-483, 2017.

14. Feng Z, Xu W, Zhang C, Liu M and Wen H: Inhibition of gammasecretase in Notch1 signaling pathway as a novel treatment for ovarian cancer. Oncotarget 8: 8215-8225, 2017.

15. Schröder N and Gossler A: Expression of Notch pathway components in fetal and adult mouse small intestine. Gene Expr Patterns 2: 247-250, 2002.

16. Sander GR and Powell BC: Expression of notch receptors and ligands in the adult gut. J Histochem Cytochem 52: 509-516, 2004.

17. Stanger BZ, Datar R, Murtaugh LC and Melton DA: Direct regulation of intestinal fate by Notch. Proc Natl Acad Sci USA 102: 12443-12448, 2005.

18. van Es JH, van Gijn ME, Riccio O, van den Born M, Vooijs M, Begthel H, Cozijnsen M, Robine S, Winton DJ, Radtke F, et al Notch/gamma-secretase inhibition turns proliferative cells in intestinal crypts and adenomas into goblet cells. Nature 435 : 959-963, 2005 .

19. Li J, Chen H, Wu S, Cheng Y, Li Q, Wang J and Zhu G: MPP(+) inhibits mGluR1/5-mediated long-term depression in mouse hippocampus by calpain activation. Eur J Pharmacol 795: 22-27, 2017.

20. Artavanis-Tsakonas S, Rand MD and Lake RJ: Notch signaling: Cell fate control and signal integration in development. Science 284: 770-776, 1999

21. Ulvklo C, MacDonald R, Bivik C, Baumgardt M, Karlsson D and Thor S: Control of neuronal cell fate and number by integration of distinct daughter cell proliferation modes with temporal progression. Development 139: 678-689, 2012.

22. Fre S, Huyghe M, Mourikis P, Robine S, Louvard D and Artavanis-Tsakonas S: Notch signals control the fate of immature progenitor cells in the intestine. Nature 435: 964-968, 2005.

23. Chu D, Wang W, Xie H, Li Y, Dong G, Xu C, Chen D, Zheng J, Li M, Lu Z, et al: Notch-1 expression in colorectal carcinoma determines tumor differentiation status. J Gastrointest Surg 13: 253-260, 2009.

24. Fabbri G, Holmes AB, Viganotti M, Scuoppo C, Belver L, Herranz D, Yan XJ, Kieso Y, Rossi D, Gaidano G, et al: Common nonmutational NOTCH1 activation in chronic lymphocytic leukemia. Proc Natl Acad Sci USA 114: E2911-E2919, 2017.

25. Fujiki K, Inamura H, Miyayama T and Matsuoka M: Involvement of Notch1 signaling in malignant progression of A549 cells subjected to prolonged cadmium exposure. J Biol Chem 292 7942-7953, 2017

26. Feng HB, Wang J, Jiang HR, Mei X, Zhao YY, Chen FR, Qu Y, Sai K, Guo CC, Yang QY, et al: $\beta$-Elemene selectively inhibits the proliferation of glioma stem-like cells through the downregulation of Notch1. Stem Cells Transl Med 6: 830-839, 2017.

27. Wieland E, Rodriguez-Vita J, Liebler SS, Mogler C, Moll I, Herberich SE, Espinet E, Herpel E, Menuchin A, Chang-Claude J, et al: Endothelial Notch1 activityf acilitates metastasis. Cancer Cell 31: 355-367, 2017.
28. Zhou YF, Sun Q, Zhang YJ, Wang GM, He B, Qi T, Zhou Y, Li XW, Li S and He L: Targeted inhibition of Notch1 gene enhances the killing effects of paclitaxel on triple negative breast cancer cells. Asian Pac J Trop Med 10: 179-183, 2017.

29. Kaka AS, Nowacki NB, Kumar B, Zhao S, Old MO, Agrawal A, Ozer E, Carrau RL, Schuller DE, Kumar P, et al: Notch-1 overexpression correlates to improved survival in cancer of the oropharynx. Otolaryngol Head Neck Surg 156: 652-659, 2017.

30. Moore CB, Guthrie EH, Huang MT and Taxman DJ: Short hairpin RNA (shRNA): Design, delivery, and assessment of gene knockdown. Methods Mol Biol 629: 141-158, 2010.

31. Han P, Fan J, Liu Y, Cuthbertson AG, Yan S, Qiu BL and Ren S: RNAi-mediated knockdown of serine protease inhibitor genes increases the mortality of Plutella xylostella challenged by destruxin A. PLoS One 9: e97863, 2014.

32. Aleksic T and Feller SM: Gamma-secretase inhibition combined with platinum compounds enhances cell death in a large subset of colorectal cancer cells. Cell Commun Signal 6: 8, 2008.

33. Radhakrishnan SK, Feliciano CS, Najmabadi F, Haegebarth A, Kandel ES, Tyner AL and Gartel AL: Constitutive expression of E2F-1 leads to p21-dependent cell cycle arrest in S phase of the cell cycle. Oncogene 23: 4173-4176, 2004.

34. Sjölund J, Johansson M, Manna S, Norin C, Pietras A, Beckman S, Nilsson E, Ljungberg B and Axelson $\mathrm{H}$ : Suppression of renal cell carcinoma growth by inhibition of Notch signaling in vitro and in vivo. J Clin Invest 118: 217-228, 2008.

35. Yi T, Baek JH, Kim HJ, Choi MH, Seo SB, Ryoo HM, Kim GS and Woo KM: Trichostatin A-mediated upregulation of p21(WAF1) contributes to osteoclast apoptosis. Exp Mol Med 39: 213-221, 2007.

36. Zoli W, Ulivi P, Tesei A, Fabbri F, Rosetti M, Maltoni R, Giunchi DC, Ricotti L, Brigliadori G, Vannini I, et al: Addition of 5-fluorouracil to doxorubicin-paclitaxel sequence increases caspase-dependent apoptosis in breast cancer cell lines. Breast Cancer Res 7: R681-R689, 2005.

37. Hasenjäger A, Gillissen B, Müller A, Normand G, Hemmati PG, Schuler M, Dörken B and Daniel PT: Smac induces cytochrome $c$ release and apoptosis independently from $\mathrm{Bax} / \mathrm{Bcl}-\mathrm{x}(\mathrm{L})$ in a strictly caspase-3-dependent manner in human carcinoma cells. Oncogene 23: 4523-4535, 2004.

38. Karst AM, Dai DL, Martinka M and Li G: PUMA expression is significantly reduced in human cutaneous melanomas. Oncogene 24: 1111-1116, 2005.

39. Stylianou S, Clarke RB and Brennan K: Aberrant activation of notch signaling in human breast cancer. Cancer Res 66 $1517-1525,2006$

This work is licensed under a Creative Commons Attribution-NonCommercial-NoDerivatives 4.0 International (CC BY-NC-ND 4.0) License. 